\title{
Research Article \\ Effects of Early MSC Intervention on Preventing the Streptozotocin-Induced T1DM Progression in Mice
}

\author{
Lanlan Zha, ${ }^{1,2}$ Xue Li $\mathbb{D}^{2},{ }^{2}$ Xiangyu Meng $\mathbb{D},{ }^{1,2}$ Yuanlin Liu $\mathbb{D},{ }^{2}$ Weijiang Liu, ${ }^{2}$ Yang Wang $\mathbb{D},{ }^{2}$ \\ Yue Fan $\mathbb{D},{ }^{2}$ Bin Zhou, ${ }^{1,2}$ Pei Li $\mathbb{D},{ }^{1,2}$ Rongxiu Zheng $\mathbb{D}^{1}$, and Yi Zhang $\mathbb{D}^{2}$ \\ ${ }^{1}$ Department of Pediatric, Tianjin Medical University General Hospital, Tianjin 300052, China \\ ${ }^{2}$ Department of Experimental Hematology and Biochemistry, Beijing Institute of Radiation Medicine, Beijing 100850, China \\ Correspondence should be addressed to Rongxiu Zheng; rzheng@tmu.edu.cn and Yi Zhang; zhangyi612@hotmail.com
}

Received 26 April 2020; Revised 29 May 2020; Accepted 15 June 2020; Published 29 July 2020

Guest Editor: Yaozu Xiang

Copyright (c) 2020 Lanlan Zha et al. This is an open access article distributed under the Creative Commons Attribution License, which permits unrestricted use, distribution, and reproduction in any medium, provided the original work is properly cited.

\begin{abstract}
Type 1 diabetes mellitus is characterized by progressive pancreatic $\beta$-cells failure and progressive autoimmunity. It is difficult to diagnose T1DM and to prevent the pancreatic $\beta$-cells destruction because of the undetectable pancreatic $\beta$-cell necrosis and abnormal autoimmunity. Here, we built streptozotocin-induced T1DM mouse model and performed MSC injection at the early stage of T1DM. We found that MSC infusion displayed enhanced effects on reducing the pathological damage and improving the survival quality. Moreover, the delivery of MSCs inhibited Th1 cell polarization and downregulated the Th1 subset ratio. The immunomodulatory mechanism of MSC was further investigated. Real-time PCR and ELISA assays demonstrated that IFN- $\gamma$ expression at both mRNA and protein level in MSC infusion T1DM mice was downregulated, partially regulated by MSC-exosome-derived miR-148a-3p. Taken together, this early therapeutic strategy may improve the clinical efficacy of MSC-based therapy in T1DM.
\end{abstract}

\section{Introduction}

Diabetes mellitus (DM) and its complications have become one of the major threats to public health nowadays. Both type 1 and type 2 diabetes result from progressive pancreatic $\beta$-cell failure. Type 1 diabetes mellitus (T1DM) is often diagnosed during childhood or adolescence. T1DM is thought to be caused by progressive autoimmunity [1] and associated with obesity and metabolic disorder [2]. The defective immune disorder results in the destruction of pancreatic $\beta$-cells, and consequently, patients with T1DM remain life span insulin-dependent. Frequently, early T1DM is difficult to be diagnosed in time because of the undetectable pancreatic $\beta$-cells necrosis and abnormal autoimmunity [3]. Hence, it is of great importance to balance the immune system for T1DM prevention [4].

The abnormal autoimmunity results in the infiltration of various immune cells into pancreatic $\beta$-cells and thus initiates the secretion of proinflammatory cytokines such as IL- $1 \beta$, TNF- $\alpha$ and IFN- $\gamma$ [5-7]. The subsequent inflammatory cytokines storm and progressive autoimmunity destroy the pancreatic $\beta$-cells, and consequently, patients with T1DM remain insulin-dependent for their life span. T1DM results in series of complications such as nephropathy, retinopathy, cardiovascular diseases, infections, and even depression $[8,9]$. Thus, intervention in the autoimmune initiation plays a pivotal role in T1DM prevention and contributes to the progression and outcome of T1DM.

Mesenchymal stem cells (MSCs) are adult stem cells and have been successfully isolated from a variety of tissues [10]. The multilineage differentiation potentials, tissue restoration capabilities, and immunoregulation properties of MSC have made it a promising stem cell source for clinical cell therapy [11]. MSC therapy has been studied in clinical trials for the treatment of acute graft-versus-host disease (aGvHD), systemic lupus erythematosus (SLE), and inflammatory bowel disease, strongly arguing for the potential application in curing autoimmune diseases including T1DM and T2DM 
$[12,13]$. Over the past decades, great efforts have been made to clarify the feasibility, safety, and efficacy of MSC. However, the complicated products and diverse origins of MSCs limit the clinical application.

Early studies in MSC have revealed that MSCs secrete soluble factors such as PGE2, iNOS, IDO, and miRNAs for long-range immunoregulatory effects $[14,15]$. Recent work has uncovered that MSC-derived extracellular vesicles (EVs), including exosomes and microvesicles (MV), are involved in cell-cell communication, cell signaling, and altering cell or tissue metabolism $[16,17]$. In the study of MSC therapies, Shao found that MSC-derived exosome shows better therapeutic effect than MSCs for cardiac repair in a rat myocardial infarction model [18], while Nojehdehi observed the immunomodulatory effects of MSC-derived exosome on experimental T1DM [17]. Thus, to uncover the molecular mechanisms of MSC's immunoregulation has far-deep implications for the T1DM prevention and therapy [19-21].

Here, we built a treptozotocin (STZ)-induced T1DM mouse model [22-25] and found that early MSC intervention could reduce the pathological damage caused by T1DM. We present both in vivo and in vitro evidence that MSC infusion upregulates the serum miR-148a-3p via secreting exosomes. MiR-148a-3p inhibits the expression of mFasl and mIFN- $\gamma$, and delays the initiation of the autoimmune and inflammatory response, thus contributes to preventing the T1DM progression. Our data demonstrated that MSC-exosome-derived miR-148a-3p displayed immune-regulatory effects, and early MSC intervention could prevent T1DM progression.

\section{Materials and Methods}

2.1. Mice. Six-week-old C57BL/6 $\left(\mathrm{H} 2^{\mathrm{b}}\right)$ female mice were purchased from SPF (Beijing) Biotechnology Co., Ltd. (http://www.spf-tsinghua.com/). All procedures and experiments performed in this study followed the Academy of Military Medical Sciences Guide for Laboratory Animals (IACUC-AMMS-017-2016-003). The mice were divided into different groups at random.

2.2. STZ-Induced Murine T1DM Model and MSC Prevention Experimental Designs. Six-week-old mice of the MSC prevention group (MP) and the diabetes mellitus group (DM) underwent STZ-induced T1DM according to the following protocol. Briefly, STZ (Sigma-Aldrich) was dissolved in Citrate Buffer Solution $(0.05 \mathrm{M}, \mathrm{pH}=4.2 \sim 4.4)$ and injected intraperitoneally $(50 \mathrm{mg} / \mathrm{kg} / \mathrm{d})$ within $15 \mathrm{~min}$ of dissolution for 5 consecutive days. The murine bone-debris-derived MSCs (as described in Supplementary Material 1) were injected intravenously at $5 \times 10^{5}$ to the MSC prevention group (MP) mice on the $7^{\text {th }}$ and $8^{\text {th }}$ days, respectively. The mice of blank control group (BC) and diabetes mellitus group (DM) received PBS buffer solution without MSCs correspondingly.

2.3. Body Weight, Blood Glucose, and Serum IFN- $\gamma$ and TNF- $\alpha$ Monitoring. Monitoring of the mice body weight and the blood glucose level was determined at $4 \sim 6$ p.m. weekly. The STZ-induced mouse T1DM model was deter- mined successfully built up with the blood glucose level greater than $16.7 \mathrm{mmol} / \mathrm{L}$ within three successive days, from the $12^{\text {th }}$ to the $14^{\text {th }}$ day.

On the $56^{\text {th }}$ day of the experiment, the serum IFN- $\gamma$ and TNF- $\alpha$ concentration were determined according to the reagent protocols of the quantitative determination kit (eBioscience).

2.4. Exosomes Isolation and Identification. MSCs were isolated from the bone debris of 1-week-old C57BL/6 mice and characterized according to Supplementary Material 1 and Supplementary Material 2 [26]. The conditional medium was collected for exosome isolation and identification. Briefly, the conditional medium was collected after culturing MSCs for 72 hours. The culture medium was centrifuged to remove the death cells and the cell debris. Then the supernatant was ultracentrifugation at 100,000 g for 4 6 hours at $4^{\circ} \mathrm{C}$. TSG101 (Abcam) and CD63 (Abcam) were used to detect the exosomes in the precipitation via Western blotting. The particle concentration and the size distribution of isolated exosomes were determined through NanoSight analysis (Particle Metrix, Meerbusch, Germany). Transmission electron microscopy was used to observe the morphology of exosomes (Hitachi H7500 TEM, Tokyo, Japan).

2.5. Histological Preparation and H\&E, IHC, and Masson's Trichrome Staining Assessments. Mice of each group were sacrificed at the end of experiments, and the kidneys were dissected for Masson's trichrome stain (TRI) to assess the diabetes complications. The islets were dissected for $\mathrm{H} \& \mathrm{E}$ and insulin-labeled insulin staining for assessment of the islets morphology and area.

2.6. miRNAs and mRNA Determination via Quantitative Real-Time PCR. Total RNA for qRT-PCR was extracted from the kidneys, spleen lymphocytes, and MSC-derived exosomes. The qRT-PCR primers were designed as shown in Supplementary Material 3. Procedure of mature miRNAs quantification was carried out using miRNA probes. Briefly, $2 \mu \mathrm{g}$ of total RNA is reverse-transcribed to cDNA using MMLV reverse transcriptase (Takara Biomedical Technology (Beijing) Co., Ltd) and a stem-loop RT primer (Sangon Biotech (Shanghai) Co., Ltd.). The reverse-transcribed cDNA was performed real-time PCR using a TaqMan PCR kit on an Applied Biosystems 7300 Sequence Detection System (Applied Biosystems). The stem-loop RT primers were designed as shown in Supplementary Material 4.

2.7. Flow Cytometry Analysis. Mice of each group were sacrificed at the end of the experiments, and the spleen was collected for lymphocytes isolation. The isolated lymphocytes were stimulated with the Cell Stimulation Cocktail and Protein Transport Inhibitors (eBioscience) for $6 \sim 18 \mathrm{~h}$. The cells were fixed and permeabilized using the BD IntraSure Kit and labeled with CD3, CD4, and IFN- $\gamma$ (eBioscience).

2.8. Luciferase Activity Assay. The wild-type $3^{\prime}$ UTR of mIFN $-\gamma$ and mFasl sequence were cloned into psiCHECK-2 and cotransfected with miR-148a-3p mimics to HEK293 


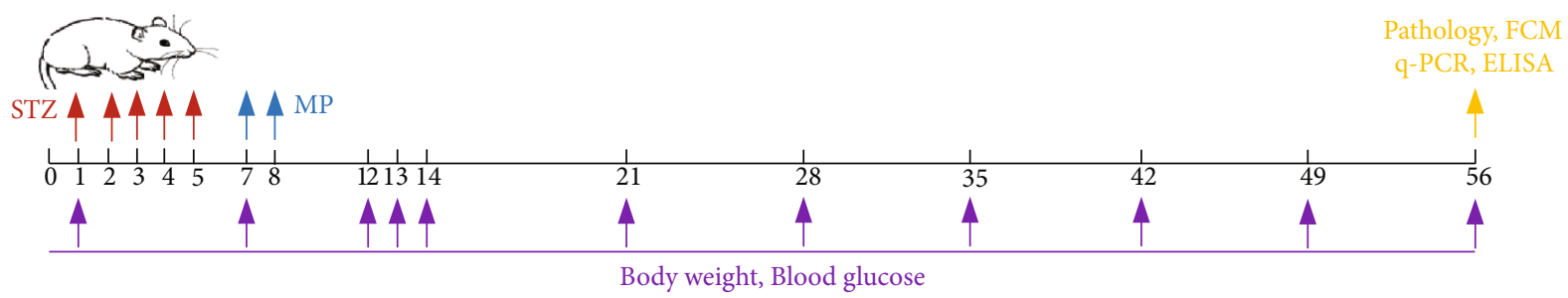

(a)

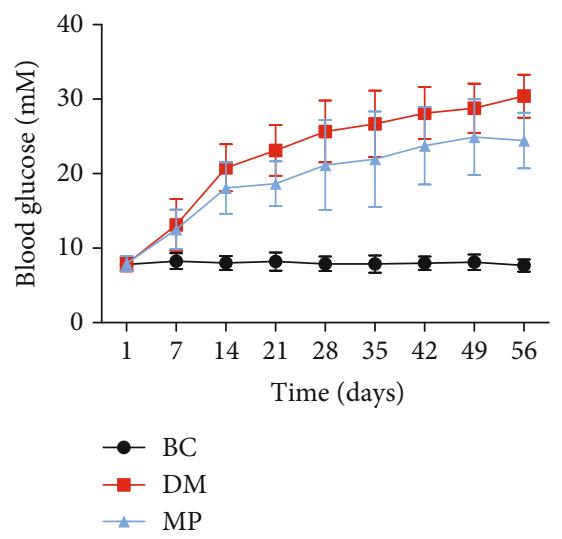

(b)

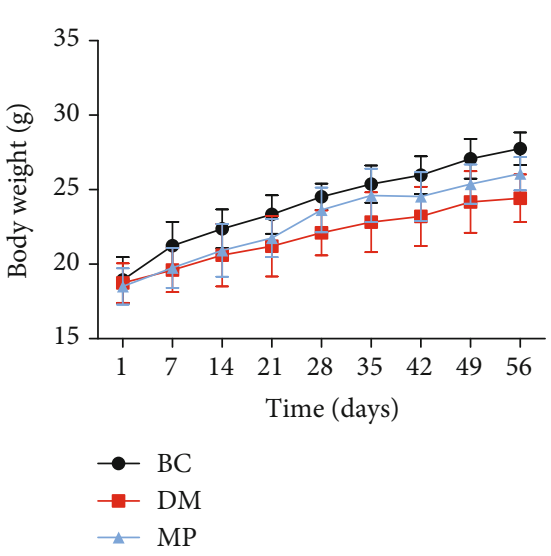

(c)

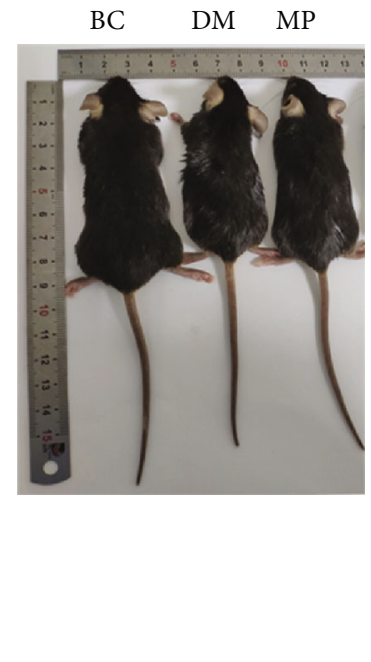

(d)

FIGURE 1: Mesenchymal stem cell infusion improves the survival quality of T1DM mice. The experimental timeline is shown in (a). The blood glucose curves and the weight growth curves are shown in (b) and (c), respectively $(n=20)$. The mice body sizes of blank control group (BC), diabetes mellitus group (DM), and MSC prevention group (MP) are shown in (d). Statistics of the $p$ value between the MP group and DM group is shown in Supplementary Material 5.

cells, respectively. 48 hours post transfections, the firefly luciferase activities and Renilla luciferase activities were measured by a Dual-Luciferase Reporter Assay System (Promega).

2.9. Statistics. Data were statistically analyzed with the GraphPad Prism software and are presented as means \pm standard errors of the mean. We performed unpaired, 2tailed Student's $t$-tests for statistical analysis, and $* p<0.05$ was deemed significant.

\section{Results}

3.1. MSC Infusion at the Early Stage of T1DM Attenuates the Progression of T1DM. We generated STZ-induced T1DM mice model and monitored the blood glucose level and body weight of each group. The experimental timeline is shown in Figure 1(a). The general condition of mice including their body size, body weight, and blood glucose level was monitored. Compared with the mice of the diabetes mellitus group (DM), MSC infusion reduced the blood glucose level of T1DM mice in the MP group (Figure 1(b)). Additionally, the slow gain in weight caused by hyperglycemia was controlled by MSC infusion. The average body weight of the MP group is larger than that of the DM group from the $14^{\text {th }}$ day till the end of the experiment (Figure 1(c)). Furthermore, the T1DM mice that received MSC prevention (MP) showed better body size, similar to the blank control group (BC) (Figure 1(d)). It should be noted that MSC infusion at early stage of T1DM maintained the blood glucose homeostasis, relieved the growth inhibition caused by hyperglycemia, and exhibited significant prevention effects.

To further determine whether MSC infusion could reduce the pathological damage to T1DM mice, we conducted histopathological examination of the mouse islets via $H \& E$ and IHC staining. As shown in Figures 2(a) and 2(b), islets of MP group exhibit mild inflammation and increased islet areas with preserved islet morphology, indicating that MSC infusion at early stage of T1DM could prevent the destruction of islets and delay the progression of T1DM. Besides, the deterioration of renal function was detected by Masson's trichrome staining, and we observed that MSC infusion helped attenuate the progression of glomerular fibrosis (arrowheads in Figure 2(c)) and maintained the glomerular structural integrity (arrows in Figure 2(c)). Moreover, the expression of TGF- $\beta 1$ and Collagen I were downregulated in the MP group (Figures 2(d) and 2(e)), suggesting that MSC infusion prevents the deterioration of renal function and thus contributes to preventing T1DM progression.

3.2. MSC Infusion Downregulates Th1 Polarization and Attenuates the Inflammatory Response. Recent studies have 

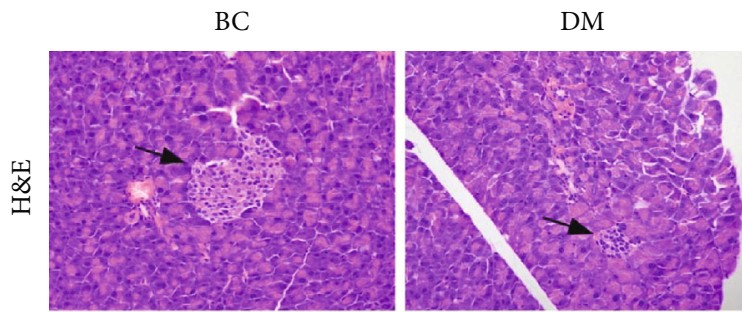

(a)
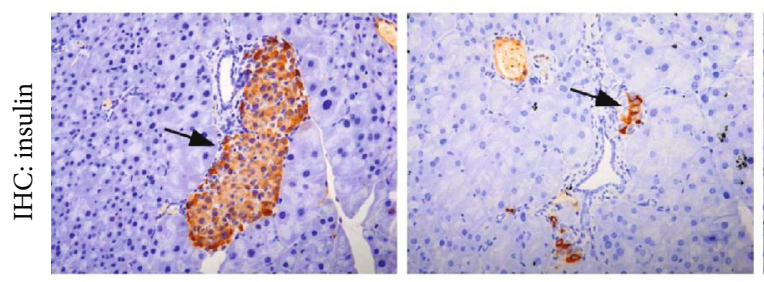

(b)
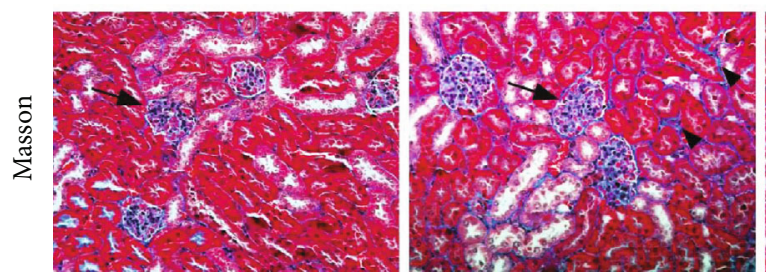

(c)
MP
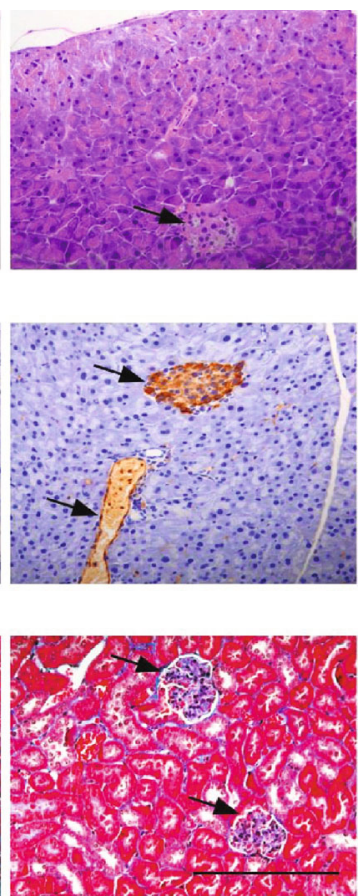

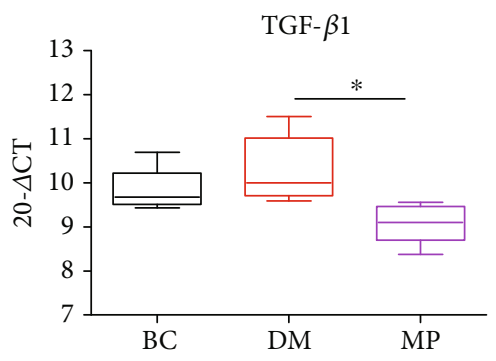

(d)

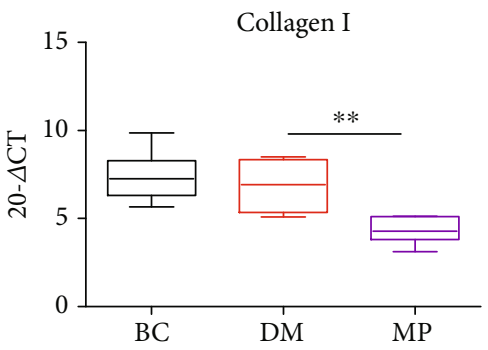

(e)

FIGURE 2: Mesenchymal stem cell infusion reduces the pathological damage to T1DM mice. H\&E staining (a) and IHC staining (b) of islets on the $56^{\text {th }}$ day of each group. Masson's trichrome staining of the glomerulus on the $56^{\text {th }}$ day of each group (c). The quantitative real-time PCR of TGF- $\beta 1$ expression (d) and Collagen I expression (e) in the kidney. Scale bars: (a-c) $200 \mu \mathrm{m}$.

reported that the polarization of $\mathrm{T}$ cell subpopulations is tightly involved in the pathological damage in T1DM. Considering the pivotal role of T cells in T1DM progression, we analyzed the spleen lymphocytes subsets via FACS. As shown in Figures 3(a)-3(c), we observed a significant reduction of the Th1 subset ratio in the MP group compared with that in the DM group. Consistent with the statistical results of the Th1 subset ratio (Figure 3(d)), the qRT-PCR results indicated a reduced expression of the proinflammatory cytokine IFN- $\gamma(p=0.0352$, Figure 3(e)). In addition, serum ELISA detecting the IFN- $\gamma$ concentration supported the idea that MSC infusion could downregulate the Th1 polarization $\left(p=0.0084\right.$, Figure $\left.3\left(e^{\prime}\right)\right)$.

We further detected the influence of MSC infusion on the inflammatory process of T1DM, and found the expression of TNF- $\alpha$ in spleen lymphocytes was reduced according to qRT-PCR $(p=0.0193$, Figure 3(f)). Similarly, the level of serum TNF- $\alpha$ was also downregulated in the MP group $\left(p=0.0175\right.$, Figure $3\left(f^{\prime}\right)$ ), indicating that MSC infusion could inhibit the expression of proinflammatory cytokines and thus attenuate the immune response. This data suggests that MSC infusion could prevent the T1DM progression via attenuating the inflammatory response.

3.3. MSC-Exosome-Derived miR-148a-3p Downregulates the Expression of $m I F N-\gamma$ and $m F a s l$. Given that MSCs secrete soluble factors such as PGE2, iNOS, IDO and miRNAs for long-range immunoregulatory effects, we speculated that there are factors regulating Th1 polarization progression.
Thus, we analyzed the conditioned culture medium of MSCs. Surprisingly, we found plenty of microvesicles with the diameter around 100 nanometers (Figures 4(a) and 4(b)) and coated with CD63 and TSG101 (Figure 4(c)), indicating that MSCs produce and secret plenty of exosomes into the culture medium.

Exosomes have been reported as the principal therapeutic reagent that mediates the immunoregulatory effects of MSCs. To investigate the role of MSC-derived exosomes in attenuating inflammatory response, we conducted microRNA screen and detected the serum level of target miRNAs. Upon our screen, we found serum miR-148a-3p was significantly upregulated when MSC was injected into the STZ-induced T1DM mice (Figure 4(d)). Moreover, bioinformatics analysis indicated that miR-148a-3p regulated the expression mIFN- $\gamma$ and $\mathrm{mFasl}$ (Figure 4(e)), which is highly conserved between mouse and human. Then, we cloned the $3^{\prime} \mathrm{UTR}$ of mIFN- $\gamma$ and mFasl into psiCHECK-2 and detected the luciferase signal. As shown in Figure 4(f), cotransfection of miR148a-3p downregulated the expression of mIFN $-\gamma$ and mFasl significantly $\left(p_{\mathrm{mIFN}-\gamma}=0.0019, p_{\mathrm{mFasl}}=0.0008\right)$. These data suggest that MSC-exosome-derived miR-148a-3p attenuates the inflammatory response initiation via inhibiting the expression of mIFN- $\gamma$ and mFasl.

\section{Discussion}

T1DM resulting from abnormal autoimmunity and pancreatic $\beta$-cells failure is a global health issue. Hence, prevention 

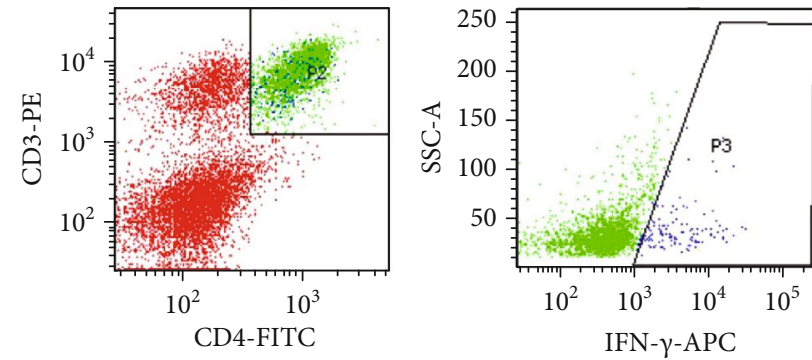

(a)

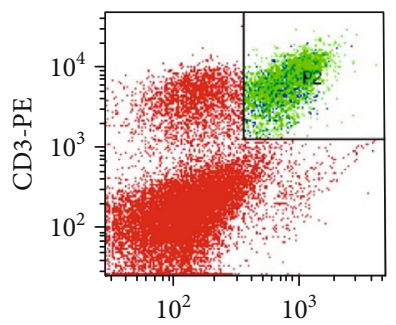

CD4-FITC

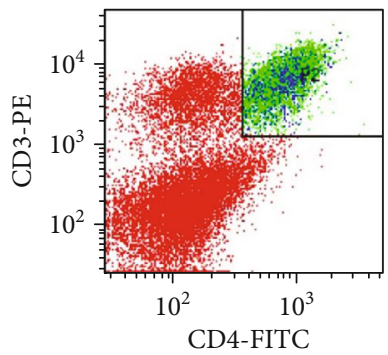

CD4-FITC

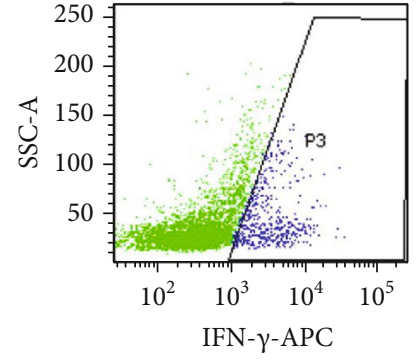

(b)

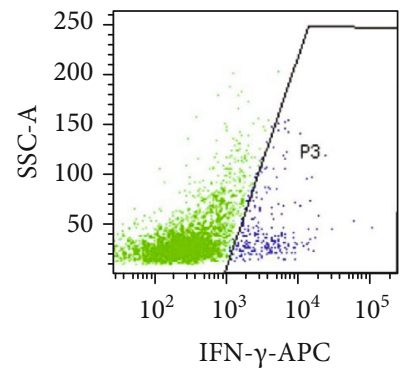

(c)

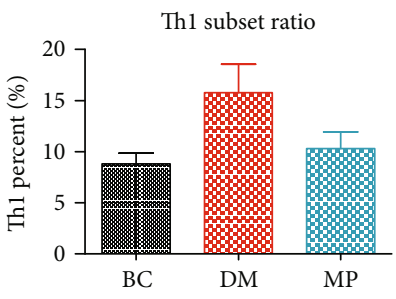

(d)

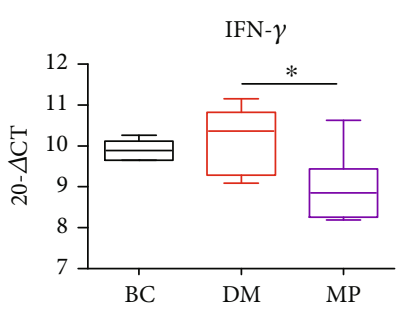

(e)

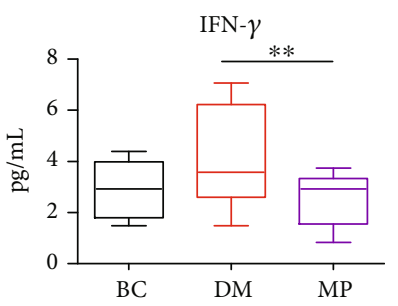

$\left(e^{\prime}\right)$

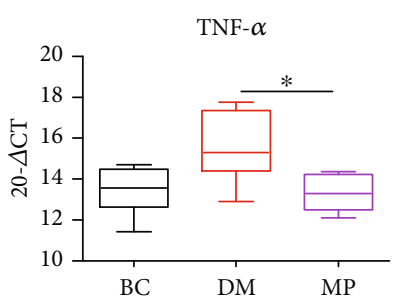

(f)

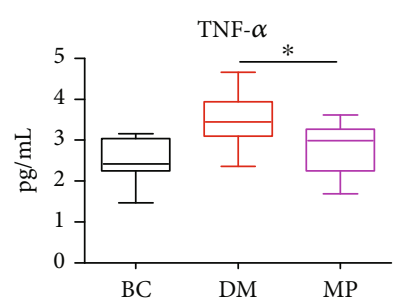

$\left(\mathrm{f}^{\prime}\right)$

Figure 3: MSC infusion inhibits Th1 polarization. On the $56^{\text {th }}$ day of the experiment, spleen lymphocytes were harvested, and the Th1 subset ratio was detected by fluorescence-activated cell sorting (FACS) analysis. Gated on CD3 and CD4, the frequencies of Th1 cells of the NC, DM, and MP groups are shown in (a-c), and statistics is shown in (d). (e) and (f) exhibit the quantitative real-time PCR detecting the expression of IFN $-\gamma$ and TNF- $\alpha$. $\left(\mathrm{e}^{\prime}\right)$ and $\left(\mathrm{f}^{\prime}\right)$ show the serum concentration of IFN- $\gamma$ and TNF- $\alpha$.

and intervention at early stage of T1DM are critical for T1DM therapy. Early diagnosis and prediction contribute to the progression and outcome of T1DM; recent work has identified IGF1, islet-specific CD8+ T cells and miRNAs as potential biomarkers for T1DM prediction [27-29]. In the current study, we found that MSC infusion at the early stage of T1DM could alleviate the STZ-induced T1DM symptoms and signs, and could improve the survival quality of T1DM mice. We conducted further experiments to clarify the effects and molecular mechanism by which MSCs prevent the progression of T1DM.

STZ specifically induces the pancreatic $\beta$-cells failure and initiates the progressive autoimmunity. The Th1 subset and the proinflammatory cytokines play pivotal roles in the inflammatory response initiation [30]. Upon our work, MSC was injected into the STZ-induced mice at the very beginning of T1DM, and we observed reduced Th1 subset ratio and relieved immune response, noting that MSC infusion could inhibit Th1 polarization and could attenuate the inflammatory response and T1DM progression.

The early intervention in T1DM requires powerful capability of immunosuppression, since STZ specifically induces the pancreatic $\beta$-cells destruction and initiates the progressive autoimmunity. MSC is a promising immunoregulator because of its capability to produce diverse immunomodulatory molecules for long-range immunoregulation [14, 15]. However, MSC therapy has been proposed for decades but has been limited for its complicated products and diverse origins. There is accumulating evidence suggesting the bidirectional immunoregulatory effects of MSC. MSCs have been confirmed to promote proliferation and metastasis in various tumors, including osteosarcoma, hepatocellular carcinoma, and ovarian cancer [31-33]. The controversial immunoregulatory effects of MSC may result from the diverse tissuespecific origins of MSC or the tissue-repair effects of MSC in a different microenvironment. Recent studies revealed that iPSC-MSCs rescue anthracycline-induced cardiomyopathy through mitochondrial transferring and paracrine actions $[34,35]$, supporting the tissue-repair effects and immunoregulatory effects of MSCs.

MSC is a promising immunoregulator because of its powerful immunoregulatory capability. Thus, it is plausible to speculate that there are factors produced by MSCs regulating Th1 polarization to prevent T1DM. Exosome secreted by 


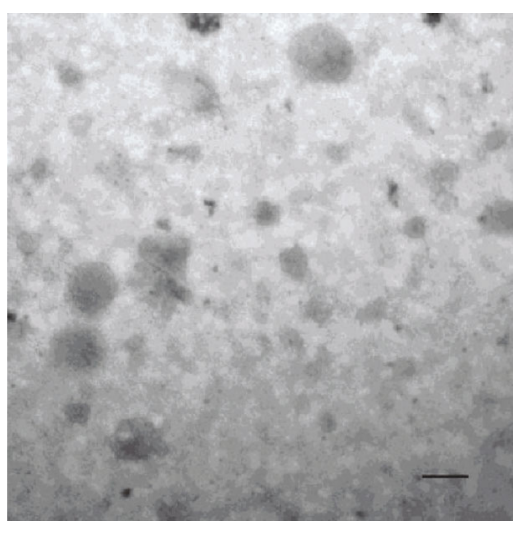

(a)

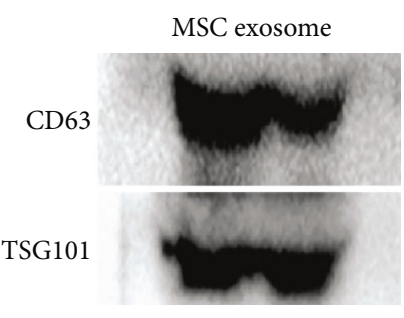

(c)

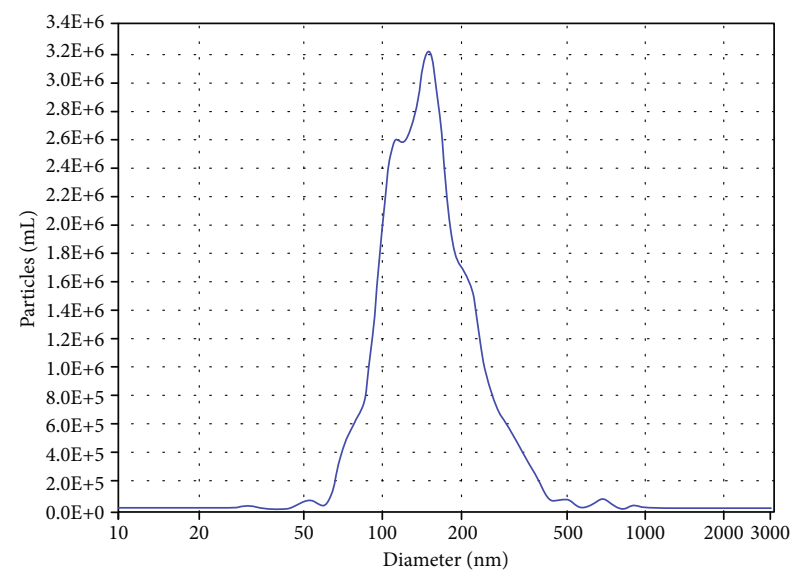

(b)

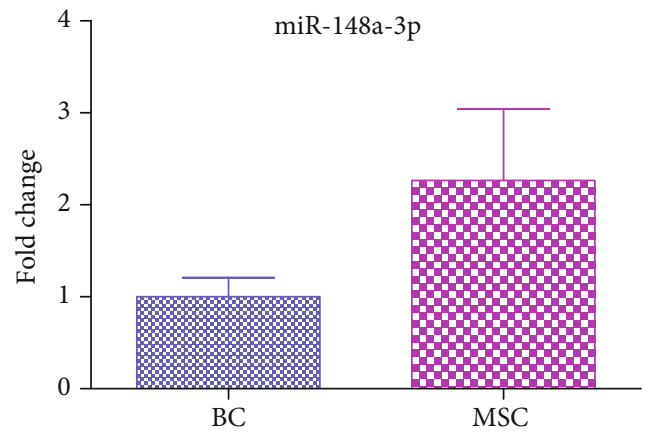

(d)

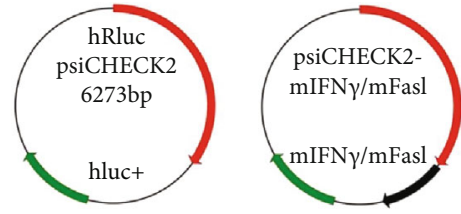

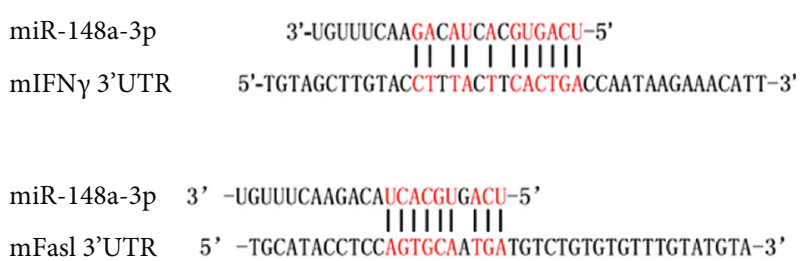

(e)

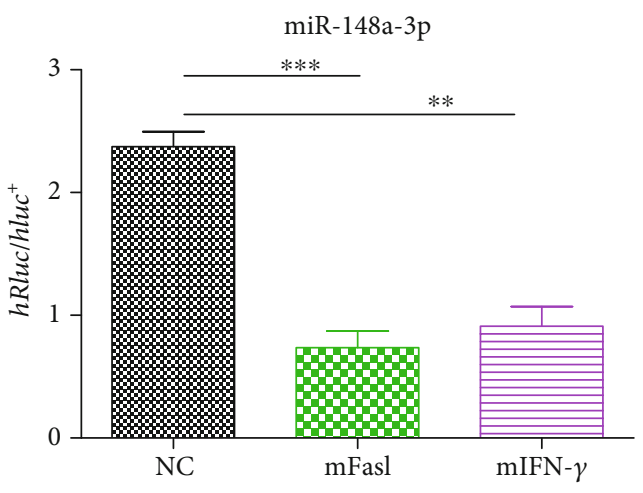

(f)

FIGURE 4: MSC-exosome-derived miR-148a-3p attenuates the inflammatory response initiation. Characterization of MSC-derived exosomes: the exosome morphology (a, scale bars: $100 \mathrm{~nm}$ ), and the exosome concentration and size distribution (b), and Western blotting of the isolated exosome labeled with TSG101 and CD63 (c). The serum level of miR148a-3p of the MP group and none-MSC injection group, $72 \mathrm{~h}$ after MSC injection (d). The bioinformatics predictions of the base matching (e) and Luciferase reporter assay (f).

MSC is a trafficking machinery for long-distance cargo transportation, and MSC-derived exosome is potential for cell-free therapy [16]. Plenty of miRNAs are packaged into exosomes and transported to the target tissues or cells via circulation for further biological function [36-38]. Upon our serum miRNAs screen and bioinformatics analysis [39-41], we identified highly conserved miR-148a-3p as a potential regulator of T1DM via inhibiting the expression of mIFN- $\gamma$ and mFasl. Previous studies have identified exosomes as diagnostic and prognostic biomarkers for T1DM [29], and the endogenous serum miR-148a-3p level is related to diabetes mellitus [42]. Thus, we conducted aforementioned experiments and have provided compelling evidence that exogenous MSCs secrete exosomes and release miR-148a-3p into the serum, which inhibits the expression of proinflammatory cytokines and thus alleviates the T1DM symptoms and signs, providing a hint that early MSC intervention and the MSC-exosome-derived miR-148a-3p could prevent the progression of T1DM. 


\section{Conclusions}

In summary, MSC intervention at early stage of T1DM could reduce the pathological damage and prevent the T1DM progression in STZ-induced T1DM mice. The exogenous MSCs secrete exosomes and release miR-148a-3p into the serum. MiR-148a-3p inhibits the expression of mFasl and mIFN- $\gamma$. Then, the Th1 polarity and the production of proinflammatory cytokines were downregulated. Thus, the early MSC intervention contributes to attenuating the inflammatory response and shows T1DM prevention effects, shedding light on the T1DM prevention strategies. However, this study only identified miR-148a-3p as a regulator in T1DM progression. The molecular mechanisms underlying the MSC-exosomemiRNA-mediated immunoregulation still require further investigation.

\section{Data Availability}

The data used to support the findings of this study are available from the corresponding author upon request.

\section{Conflicts of Interest}

The authors declare that there is no conflict of interest regarding the publication of this paper.

\section{Authors' Contributions}

Lanlan Zha, Xue Li, and Xiangyu Meng contributed equally to this work.

\section{Acknowledgments}

This work was supported by grants from the National Key Research and Development Program of China (2016YFC1000305), Key Projects of Tianjin Natural Science Foundation (17JCZDJC36400), Key Research Project of Tianjin Municipal Commission of Health (16KG123), and Science and Technology Popularization Project of Tianjin Science and Technology Bureau (18KPHDSF00140).

\section{Supplementary Materials}

Scheme S1: the methods for isolation and identification of MSCs from murine bone debris. Figure S2: the methods for isolation and identification of MSCs from murine bone debris. Table S3: the qRT-PCR primer sequence. Table S4: stem-loop RT primer sequence. Table S5: the statistics of $p$ value between MP group and DM group of Figures 1(b) and 1(c). (Supplementary Materials)

\section{References}

[1] P. Pozzilli, E. Maddaloni, and R. Buzzetti, "Combination immunotherapies for type 1 diabetes mellitus," Nature Reviews Endocrinology, vol. 11, no. 5, pp. 289-297, 2015.

[2] S. Polsky and S. L. Ellis, "Obesity, insulin resistance, and type 1 diabetes mellitus," Current Opinion in Endocrinology, Diabetes and Obesity, vol. 22, no. 4, pp. 277-282, 2015.
[3] B. O. Roep and T. I. M. Tree, "Immune modulation in humans: implications for type 1 diabetes mellitus," Nature Reviews Endocrinology, vol. 10, no. 4, pp. 229-242, 2014.

[4] C. Chen, L. Li, H. Qin et al., "Effects of Irbesartan pretreatment on pancreatic $\beta$-cell apoptosis in STZ- induced acute prediabetic mice," Oxidative Medicine and Cellular Longevity, vol. 2018, Article ID 8616194, 10 pages, 2018.

[5] H. Kolb, U. Wörz-Pagenstert, R. Kleemann, H. Rothe, P. Rowsell, and F. W. Scott, "Cytokine gene expression in the BB rat pancreas: natural course and impact of bacterial vaccines," Diabetologia, vol. 39, no. 12, pp. 1448-1454, 1996.

[6] A. Rabinovitch, W. Suarez-Pinzon, A. el-Sheikh, O. Sorensen, and R. F. Power, "Cytokine gene expression in pancreatic isletinfiltrating leukocytes of BB rats: expression of Th1 cytokines correlates with $\beta$-cell destructive insulitis and IDDM," Diabetes, vol. 45, no. 6, pp. 749-754, 1996.

[7] B. Baumann, H. H. Salem, and B. O. Boehm, "Anti-inflammatory therapy in type 1 diabetes," Current Diabetes Reports, vol. 12, no. 5, pp. 499-509, 2012.

[8] K. Reidy, H. M. Kang, T. Hostetter, and K. Susztak, "Molecular mechanisms of diabetic kidney disease," The Journal of Clinical Investigation, vol. 124, no. 6, pp. 2333-2340, 2014.

[9] P. D. O’Brien, S. A. Sakowski, and E. L. Feldman, "Mouse models of diabetic neuropathy," ILAR Journal, vol. 54, no. 3, pp. 259-272, 2014.

[10] P. Bianco, P. G. Robey, and P. J. Simmons, "Mesenchymal stem cells: revisiting history, concepts, and assays," Cell Stem Cell, vol. 2, no. 4, pp. 313-319, 2008.

[11] X. Wei, X. Yang, Z. P. Han, F. F. Qu, L. Shao, and Y. F. Shi, "Mesenchymal stem cells: a new trend for cell therapy," Acta Pharmacologica Sinica, vol. 34, no. 6, pp. 747-754, 2013.

[12] J. Cho, M. D'Antuono, M. Glicksman, J. Wang, and J. Jonklaas, "A review of clinical trials: mesenchymal stem cell transplant therapy in type 1 and type 2 diabetes mellitus," American Journal of Stem Cells, vol. 7, no. 4, pp. 82-93, 2018.

[13] W. Zhang, L. Zhou, J. Dang et al., "Human Gingiva-derived mesenchymal stem cells ameliorate streptozoticin- induced T1DM in mice via suppression of T effector cells and upregulating treg subsets," Scientific Reports, vol. 7, no. 1, article 15249, 2017.

[14] S. W. Ferguson, J. Wang, C. J. Lee et al., "The microRNA regulatory landscape of MSC-derived exosomes: a systems view," Scientific Reports, vol. 8, no. 1, article 1419, 2018.

[15] V. B. R. Konala, M. K. Mamidi, R. Bhonde, A. K. Das, R. Pochampally, and R. Pal, "The current landscape of the mesenchymal stromal cell secretome: a new paradigm for cell-free regeneration," Cytotherapy, vol. 18, no. 1, pp. 13-24, 2016.

[16] D. G. Phinney and M. F. Pittenger, "Concise review: MSCDerived exosomes for cell-free therapy," Stem Cells, vol. 35, no. 4, pp. 851-858, 2017.

[17] S. Nojehdehi, S. Soudi, A. Hesampour, S. Rasouli, M. Soleimani, and S. M. Hashemi, "Immunomodulatory effects of mesenchymal stem cell-derived exosomes on experimental type-1 autoimmune diabetes," Journal of Cellular Biochemistry, vol. 119, no. 11, pp. 9433-9443, 2018.

[18] L. Shao, Y. Zhang, B. Lan et al., "MiRNA-sequence indicates that mesenchymal stem cells and exosomes have similar mechanism to enhance cardiac repair," BioMed Research International, vol. 2017, Article ID 4150705, 9 pages, 2017. 
[19] P.-O. Carlsson, O. Korsgren, and K. Le Blanc, "Mesenchymal stromal cells to halt the progression of type 1 diabetes?," Current Diabetes Reports, vol. 15, no. 7, p. 46, 2015.

[20] J. N. U. Yaochite, C. Caliari-Oliveira, L. E. B. de Souza et al., "Therapeutic efficacy and biodistribution of allogeneic mesenchymal stem cells delivered by intrasplenic and intrapancreatic routes in streptozotocin-induced diabetic mice," Stem Cell Research \& Therapy, vol. 6, no. 1, p. 31, 2015.

[21] M. Li and S. Ikehara, "Stem cell treatment for type 1 diabetes," Frontiers in Cell and Developmental Biology, vol. 2, p. 9, 2014.

[22] L.-J. Yan and J. Wu, "Streptozotocin-induced type 1 diabetes in rodents as a model for studying mitochondrial mechanisms of diabetic \&beta; cell glucotoxicity," Diabetes, Metabolic Syndrome and Obesity: Targets and Therapy, vol. 8, pp. 181-188, 2015.

[23] E. Horwitz, L. Krogvold, S. Zhitomirsky et al., "beta-Cell DNA damage response promotes islet inflammation in type 1 diabetes," Diabetes, vol. 67, no. 11, pp. 2305-2318, 2018.

[24] X. Han, Y. Tao, Y. Deng et al., "Metformin ameliorates insulitis in STZ-induced diabetic mice," Peer Journal, vol. 5, p. e3155, 2017.

[25] A. Al-awar, K. Kupai, M. Veszelka et al., "Experimental Diabetes Mellitus in Different Animal Models," Experimental Diabetes Research, vol. 2016, Article ID 9051426, 12 pages, 2016.

[26] H. Zhu, Z. K. Guo, X. X. Jiang et al., “A protocol for isolation and culture of mesenchymal stem cells from mouse compact bone," Nature Protocols, vol. 5, no. 3, pp. 550-560, 2010.

[27] C. Greenhill, "IGFs potential biomarkers for type 1 diabetes mellitus," Nature Reviews Endocrinology, vol. 16, no. 3, pp. 130-131, 2020.

[28] F. S. Wong and L. Wen, "A predictive $\mathrm{CD}^{+} \mathrm{T}$ cell phenotype for T1DM progression," Nature Reviews Endocrinology, vol. 16, no. 4, pp. 198-199, 2020.

[29] M. Garciacontreras, R. W. Brooks, L. Boccuzzi, P. D. Robbins, and C. Ricordi, "Exosomes as biomarkers and therapeutic tools for type 1 diabetes mellitus," European Review for Medical and Pharmacological Sciences, vol. 21, no. 12, pp. 29402956, 2017.

[30] G. A. Hassan, H. A. Sliem, A. T. Ellethy, and S. Salama Mel, "Role of immune system modulation in prevention of type 1 diabetes mellitus," Indian Journal of Endocrinology and Metabolism, vol. 16, no. 6, pp. 904-909, 2012.

[31] J. Chen, T. Ji, D. Wu et al., "Human mesenchymal stem cells promote tumor growth via MAPK pathway and metastasis by epithelial mesenchymal transition and integrin $\alpha 5$ in hepatocellular carcinoma," Cell Death \& Disease, vol. 10, no. 6, p. $425,2019$.

[32] Y. Zheng, G. Wang, R. Chen, Y. Hua, and Z. Cai, "Mesenchymal stem cells in the osteosarcoma microenvironment: their biological properties, influence on tumor growth, and therapeutic implications," Stem Cell Research \& Therapy, vol. 9, no. 1, p. 22, 2018.

[33] T. Gao, Y. Yu, Q. Cong et al., "Human mesenchymal stem cells in the tumour microenvironment promote ovarian cancer progression: the role of platelet-activating factor," BMC Cancer, vol. 18, no. 1, p. 999, 2018.

[34] Y. Zhang, X. Liang, S. Liao et al., "Potent paracrine effects of human induced pluripotent stem cell-derived mesenchymal stem cells attenuate doxorubicin-induced cardiomyopathy," Scientific Reports, vol. 5, no. 1, article 11235, 2015.
[35] Y. Zhang, Z. Yu, D. Jiang et al., "iPSC-MSCs with high intrinsic MIRO1 and sensitivity to TNF- $\alpha$ yield efficacious mitochondrial transfer to rescue anthracycline-induced cardiomyopathy," Stem Cell Reports, vol. 7, no. 4, pp. 749-763, 2016.

[36] R. C. Lai, R. W. Y. Yeo, and S. K. Lim, "Mesenchymal stem cell exosomes," Seminars in Cell \& Developmental Biology, vol. 40, pp. 82-88, 2015.

[37] D. P. Sarvar, K. Shamsasenjan, and P. Akbarzadehlaleh, "Mesenchymal stem cell-derived exosomes: new opportunity in cell-free therapy," Advanced Pharmaceutical Bulletin, vol. 6, no. 3, pp. 293-299, 2016.

[38] K. Yin, S. Wang, and R. C. Zhao, "Exosomes from mesenchymal stem/stromal cells: a new therapeutic paradigm," Biomarker Research, vol. 7, no. 1, p. 8, 2019.

[39] T. S. Assmann, M. Recamonde-Mendoza, B. M. De Souza, and D. Crispim, "Micro RNA expression profiles and type 1 diabetes mellitus: systematic review and bioinformatic analysis," Endocrine Connections, vol. 6, no. 8, pp. 773-790, 2017.

[40] E. Satake, M. G. Pezzolesi, Z. I. Md Dom, A. M. Smiles, M. A. Niewczas, and A. S. Krolewski, "Circulating miRNA profiles associated with hyperglycemia in patients with type 1 diabetes," Diabetes, vol. 67, no. 5, pp. 1013-1023, 2018.

[41] M. Garcia-Contreras, S. H. Shah, A. Tamayo et al., "Plasmaderived exosome characterization reveals a distinct microRNA signature in long duration type 1 diabetes," Scientific Reports, vol. 7, no. 1, article 5998, 2017.

[42] P. de Candia, G. Spinetti, C. Specchia et al., "A unique plasma microRNA profile defines type 2 diabetes progression," PLoS One, vol. 12, no. 12, article e0188980, 2017. 